\title{
ADSORÇÃO DE ATRAZINA EM SOLO TROPICAL SOB PLANTIO DIRETO E CONVENCIONAL
}

\author{
FÁBIO VERÍSSIMO CORREIA* \\ FÁBIO MARTINS MERCANTE** \\ AMOACY CARVALHO FABRÍCIO** \\ TACIO MAURO PEREIRA DE CAMPOS*** \\ EURÍPEDES VARGAS JR*** \\ TOMAZ LANGENBACH ${ }^{* * * *}$
}

\begin{abstract}
O objetivo deste trabalho foi estudar a influência do tipo de sistema de manejo sobre o potencial de adsorção do herbicida atrazina no solo. Os resultados de coeficiente de adsorção mostram que as isotermas de adsorção de atrazina nos solos sob sistema convencional (SC), mata (SN) e solo subsuperficial (SUB) ajustaram-se ao modelo de Freundlich. Já para o plantio direto (PD), o modelo não correspondeu ao comportamento do herbicida. A adsorção aumentou em função do tempo de contado da atrazina com os solos sob PD e SN, diminuindo os riscos de lixiviação com o tempo. O fator que promoveu maior potencial de adsorção no solo sob PD não foi exclusivamente o teor de matéria orgânica (MO), mas a qualidade desse componente no solo. O maior potencial de adsorção e a dessorção nula no sistema PD indicaram maior potencial na redução da lixiviação e da contaminação dos lençóis d'água. Esses resultados recomendam o PD como tecnologia capaz de reduzir o impacto ambiental de atrazina e provavelmente de outros pesticidas.
\end{abstract}

PALAVRAS-CHAVE: POLUIÇÃO DO SOLO; LIXIVIAÇÃO; HERBICIDA; ADSORÇÃO; PLANTIO DIRETO; ATRAZINA.

\footnotetext{
* Pesquisador, Instituto de Microbiologia Professor Paulo de Góes (IMPPG), Universidade Federal do Rio de Janeiro (UFRJ), Rio de Janeiro, Brasil (e-mail: fabioverissimobr@yahoo.com.br).

** Pesquisador, Empresa Brasileira de Pesquisa Agropecuária, Centro de Pesquisa Agropecuária Oeste, Dourados, Mato Grosso do Sul (e-mail:mercante@cpao.embrapa.br, amoacy@cpao.embrapa.br).

*** Professor, Departamento de Geotecnia Ambiental, Pontifícia Universidade Católica do Rio de Janeiro (PUC-Rio), Rio de Janeiro (e-mail: tacio@civ.puc-rio.br, vargas@civ.puc-rio.br).

**** Professor Adjunto, IMPPG, UFRJ, Rio de Janeiro, Brasil (e-mail: tomazlange@yahoo.com.br).
} 


\section{INTRODUÇÃO}

A adsorção, responsável pela ligação dos agrotóxicos ao solo, influencia os processos de transporte por lixiviação, escorrimento superficial e volatilização. Representa fator preponderante na disponibilidade desses compostos químicos, pois maior ou menor adsorção define seus efeitos ecotoxicológicos, toxicológicos e sua capacidade de biodegradação.

Os pesticidas no ambiente solo apresentam-se de três formas: a) solúveis ou disponíveis na solução do solo; b) adsorvidos e em equilíbrio dinâmico com a solução do solo e; c) fortemente ligados (OECD, 2000).

A adsorção de determinada substância é definida como a relação entre sua concentração ligada nos componentes da fase sólida do solo e a que permanece dissolvida na fase líquida. Essa relação pode ser reversível ou parcialmente irreversível, afetando a atividade, a biodisponibilidade e a persistência dos pesticidas no solo. No processo chamado de dessorção, a molécula é liberada total ou parcialmente à solução do solo promovendo novo equilíbrio na solução do solo (FÜHR et al., 1998; OECD, 2000).

Dependendo da energia e do mecanismo de ligação nos colóides do solo, o pesticida pode se apresentar na forma não-disponível por estar fortemente ligado. Em sua forma não-disponível, a substância não está em equilíbrio com a solução do solo (resíduo-ligado). Quando parte do resíduoligado retorna a solução do solo, o processo é conhecido como remobilização (LAVORENTI, 1997; FÜHR et al., 1998). O retorno de um resíduo ligado à solução do solo não é tão dinâmico quanto o de um adsorvido, e depende das condições impostas pelo meio.

BLANCO e MACHADO (2000); OLIVEIRA JÚNIOR (1998); PRATA et al. (2001) descreveram a adsorção de pesticidas em sistemas de manejo convencional, porém pouco se sabe sobre o comportamento dessas moléculas em solos submetidos ao manejo de plantio direto em condições brasileiras.

A palha depositada na superfície e a decomposição da rizosfera no solo sob sistema de plantio direto influenciam os padrões de acumulação de matéria orgânica (MO) no solo, afetando suas propriedades físicas, químicas e biológicas. O teor de carbono orgânico total acumulado na superfície do solo estimula a biomassa ao longo do tempo e por conseqüência o aumento da atividade microbiana, podendo resultar no aumento do processo de degradação de pesticidas (COSTA, MONTEIRO e TORNISIELO, 1997; ENTRY e EMMINGHAM, 1995; PRATA et al., 2001). Por outro lado, foi observado que maior teor de $\mathrm{MO}$ no solo também pode promover aumento no processo de adsorção do herbicida e seus metabólitos. Tal fato os torna indisponíveis ao processo de biodegradação, aumentando assim sua persistência no ambiente (LANGENBACH, SCHROLL e SCHEUNERT, 2001).

A caracterização do grau de adsorção-dessorção de herbicidas em solos tem sido extensivamente estudada com o uso dos coeficientes de adsorção $\left(K_{d}\right)$ e de Freundlich $\left(K_{f}\right)(V A Z$, 1994; OLIVEIRA, 1998). O valor da constante $K_{d}$, em alguns trabalhos, não foi exato o suficiente para representar o processo de adsorção de pesticidas em ampla faixa de concentração, sendo necessário utilizar a isoterma de Freundlich para descrever esse fenômeno.

Neste trabalho foi estudada a influência do tipo de sistema de manejo agrícola sobre o potencial de adsorção do herbicida atrazina (produto comercial Gesaprim 500) no solo. A adsorção pode influenciar o potencial de lixiviação e assim a contaminação de águas subterrâneas por esse produto. As informações obtidas neste trabalho também serão utilizadas no teste e validação de modelos que descrevem o transporte de pesticidas no ambiente.

\section{MATERIAL E MÉTODOS}

\subsection{DESCRIÇÃO DA ÁREA}

Utilizou-se Latossolo Vermelho distroférrico típico de textura muito argilosa ( $70 \%$ de argila),

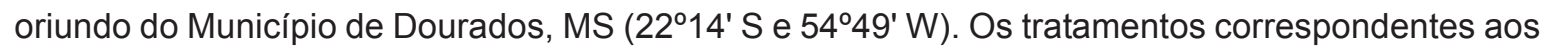


diferentes sistemas de manejo do solo foram dispostos em faixas no campo experimental da Embrapa Agropecuária Oeste, implantado desde 1995. Os sistemas intensivos de produção avaliados consistiram de manejo sob plantio convencional (aração e gradagem), sistema plantio direto e sistema natural (mata nativa) para comparação. Informações mais detalhadas dos sistemas de produção podem ser encontradas em SILVA et al. (2006). A descrição físico-química do solo foi determinada pelo Laboratório de Análise de Solo da Embrapa Agropecuária Oeste e está apresentada na Tabela 1.

\section{TABELA 1 - PARÂMETROS FÍSICO - QUÍMICOS DOS SOLOS ESTUDADOS}

\begin{tabular}{lcccccc}
\hline \multicolumn{1}{c}{ SISTEMA } & Areia & $\begin{array}{c}\text { Silte } \\
\text { \% }\end{array}$ & Argila & CTC ef. & $\begin{array}{c}\mathbf{p H} \\
\left(\mathbf{H}_{\mathbf{2}} \mathbf{O}\right)\end{array}$ & $\begin{array}{c}\mathbf{M O} \\
\mathbf{g . d m}^{-3}\end{array}$ \\
\hline Plantio direto & 10 & 11 & 79 & 7,0 & 5,0 & 33,3 \\
Plantio convencional & 9 & 4 & 87 & 18,4 & 6,2 & 59,0 \\
Sistema natural & 19 & 3 & 78 & 6,1 & 5,0 & 31,3 \\
Solo subsuperficial & 19 & 3 & 78 & 6,0 & 5,0 & 31,3 \\
\hline
\end{tabular}

CTC ef. = capacidade de troca catiônica efetiva, $\mathrm{MO}$ = matéria orgânica .

\subsection{COLETA DE AMOSTRAS DE SOLO}

Foram utilizadas no estudo, amostras da camada superficial $(0-20 \mathrm{~cm})$ das parcelas sob os sistemas de plantio direto (PD), plantio convencional (SC), solo sob mata nativa (SN) e solo subsuperficial (SUB) na profundidade de 90 a $100 \mathrm{~cm}$. A partir de amostras coletadas em 5 pontos de cada parcela foram preparadas amostras compostas para execução do experimento. As amostras foram secas ao ar e passadas em peneiras de $2 \mathrm{~mm}$ de malha (OECD, 2000).

\subsection{ADSORÇÃO DE ATRAZINA}

Pesaram-se $20 \mathrm{~g}$ de amostra de cada solo em tubos de centrífuga de $30 \mathrm{~mL}$ nos quais foram adicionados $20 \mathrm{~mL}$ de soluções de atrazina (Gesaprim 500 ) nas concentrações de 0; 0,5; 1,0; 2,5; 5,0 e 10,0 (g.mL-1 de ingrediente ativo (i.a.), preparadas em solução de $\mathrm{CaCl}_{2} \cdot 2 \mathrm{H}_{2} \mathrm{O}$ $\left(0,01\right.$ mol. $\left.\mathrm{L}^{-1}\right)$ como eletrólito-suporte. Verificou-se a concentração inicial de atrazina (Gesaprim 500 ) dessas soluções, previamente, usando cromatografia a líquido de alta eficiência (CLAE). Os tubos foram devidamente fechados e incubados com agitação orbital durante 48 horas. Logo em seguida, as amostras foram centrifugadas durante 20 minutos a $760 \mathrm{~g} \mathrm{em}$ centrífuga Beckman J2-HS. O sobrenadante foi retirado, filtrado em membrana com poro de 0,45 $\mu \mathrm{m} \mathrm{e}$ uma alíquota analisada por CLAE. Todo o experimento foi efetuado em triplicata e suas concentrações corrigidas com o branco, preparado em solução de $\mathrm{CaCl}_{2} \cdot 2 \mathrm{H}_{2} \mathrm{O} 0,01$ mol.L-1. Executou-se o experimento em temperatura de $21^{\circ} \mathrm{C}$. Em testes preliminares verificou-se $\mathrm{O}$ ponto de equilíbrio (tempo em que a concentração do herbicida na solução fica em equilíbrio com a quantidade adsorvida nas partículas do solo) nos tempos de incubação de 24, 48, 72 e $96 \mathrm{~h}$. Esses estudos evidenciaram adsorção nula do produto na parede dos tubos de centrifuga (AHMAD, KOOKAMA e ALSTON, 2001). 


\subsection{DESSORÇÃO DE ATRAZINA}

Após a retirada do sobrenadante empregado para a determinação da adsorção, adicionaramse aos tubos de centrifuga $20 \mathrm{~mL}$ de solução $0,01 \mathrm{~mol}^{-\mathrm{L}^{-1}} \mathrm{de} \mathrm{CaCl}_{2} \cdot 2 \mathrm{H}_{2} \mathrm{O}$. Os tubos foram novamente fechados e submetidos à agitação por 24 horas. Em seguida, as amostras foram centrifugadas durante 20 minutos a $760 \mathrm{~g}$, sendo o sobrenadante retirado, filtrado em membrana de 0,45 $\mu \mathrm{m}$ e uma alíquota analisada por CLAE (AHMAD, KOOKAMA e ALSTON, 2001).

\subsection{ANÁLISE POR CLAE}

A concentração de atrazina foi determinada por CLAE em equipamento Waters 600 Controller, equipado com coluna de fase reversa $\operatorname{RP}_{8}(5 \mu \mathrm{m}, 250 \times 4 \mathrm{~mm}$, LiChrospher 100, Merck) antecedida por coluna guarda $(10 \times 3 \mathrm{~mm})$. A absorção foi medida com detector de ultravioleta (UV) em $254 \mathrm{~nm}$. Efetuou-se a corrida em fase móvel de metanol + água milliq em duas concentrações (65:35/v:v, 50:50/v:v) para evitar falsos resultados decorrentes de impurezas. A coluna foi mantida em temperatura ambiente $\left(21^{\circ} \mathrm{C}\right) \mathrm{com}$ o fluxo da fase móvel de $0,5 \mathrm{~mL} \mathrm{~min}^{-1}$. Todas as amostras foram analisadas sob essas condições com o volume de injeção de $20 \mu \mathrm{L}$. A concentração de atrazina nas amostras também foi confirmada pela análise do padrão analítico, apresentando linearidade do método com curvas de coeficiente de regressão de 0,999 e limite de detecção de $0,2 \mu \mathrm{g}$. $\mathrm{L}^{-1}$ (CORREIA et al., 2007).

\subsection{CÁLCULOS DOS COEFICIENTES DE ADSORÇÃO}

Os parâmetros das Isotermas de adsorção foram calculados pela fórmula linearizada de Freundlich: $\log (x / m)=\log \left(K_{f}\right)+1 / n \log (C e)$, em que $x / m=$ a quantidade de atrazina adsorvida (mg. $\mathrm{Kg}^{-1}$ ) ou remanescente após a dessorção; $\mathrm{Ce}=$ a concentração no equilíbrio $\left(\mathrm{mg} . \mathrm{L}^{-1}\right) ; \mathrm{K}_{\mathrm{f}}=$ coeficiente de adsorção/dessorção de Freundlich e $n$ = parâmetros de regressão característicos para cada sistema solo-substância estudado (LAVORENTI, 1996; LUCHINI, 1997). O parâmetro $K_{f}$ foi analisado de acordo com a tabela descrita nas normas para avaliação de agentes químicos (IBAMA, 1990).

$\mathrm{O}$ coeficiente de distribuição $\mathrm{K}_{\mathrm{d}}$, entre a fase sólida e a solução, para as diferentes concentrações de atrazina foi calculado pela seguinte equação: $K_{d}=(x / m) / C e$. As quantidades de atrazina adsorvidas foram calculadas pela equação: $\mathrm{x} / \mathrm{m}=(\mathrm{Ci}-\mathrm{Ce}) \cdot \mathrm{V} / \mathrm{m}$, em que: $\mathrm{Ci}$ e $\mathrm{Ce}=$ concentrações de atrazina inicial e no equilíbrio $\left(m g . L^{-1}\right) ; V=$ volume da solução de atrazina $(L)$ e $\mathrm{m}=$ massa do solo $(\mathrm{kg})$. O parâmetro $\mathrm{K}_{\mathrm{d}}$ (calculado assumindo-se $\mathrm{n}=1$ ) é considerado próprio para a comparação da capacidade adsortiva entre solos (HANCE, 1988). A porcentagem de adsorção foi medida como: \% adsorvida $=100 \times(\mathrm{Ci}-\mathrm{Ce}) / \mathrm{Ci}$.

Calculou-se o parâmetro $\mathrm{K}_{\mathrm{om}}=\left(\mathrm{K}_{\mathrm{d}} \times 100\right) / \% \mathrm{MO}$, em que $\mathrm{K}_{\mathrm{om}}=$ coeficiente de adsorção normalizado em relação ao conteúdo de carbono orgânico do solo e \% $\mathrm{MO}=$ porcentagem de carbono orgânico. Segundo RAO e JESSUP (1983) esse parâmetro é mais adequado para comparação da adsorção de determinado agrotóxico em diferentes tipos de solos.

\section{RESULTADOS E DISCUSSÃO}

\subsection{ADSORÇÃO $\left(K_{F}\right)$}

As isotermas de adsorção de atrazina, plotadas graficamente (Figura 1), apresentaram coeficientes $\mathrm{K}_{\mathrm{f}}$ de 11,$28 ; 5,15 ; 2,40$; e 2,4 para os solos sob PD, SN, SC e SUB, respectivamente (Tabela 2). 


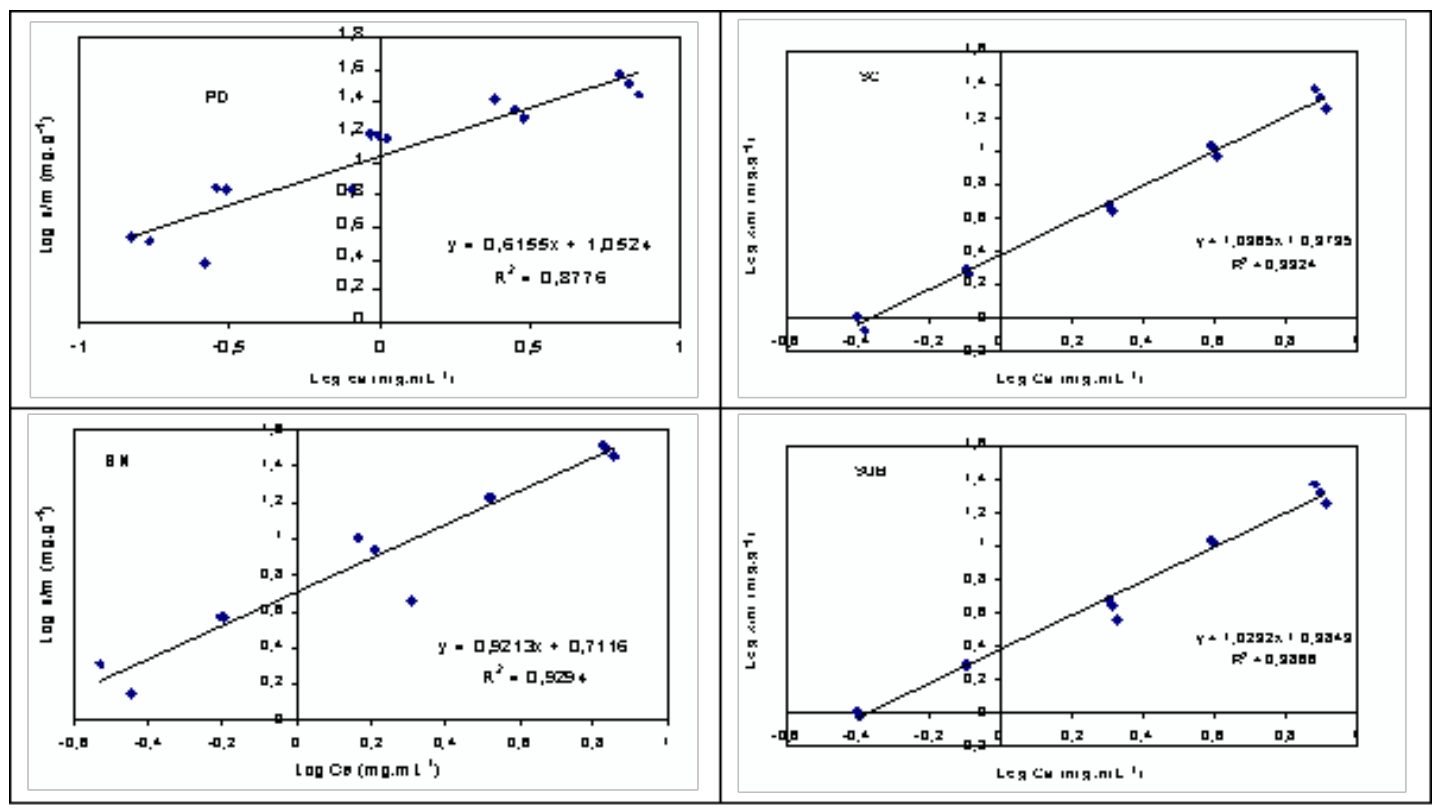

Cada ponto representa a média de três replicatas.

TABELA 2 - PARÂMETROS DAS ISOTERMAS DE ADSORÇÃO E DESSORÇÃO DOS SOLOS SOB PD, SC, SN E SUB

\begin{tabular}{|c|c|c|c|c|c|c|}
\hline & \multicolumn{3}{|c|}{ Adsorção } & \multicolumn{3}{|c|}{ Dessorção } \\
\hline SOLO & $K_{f}$ & 1/n & $r^{2}$ & $K_{f}$ & $1 / n$ & $r^{2}$ \\
\hline PLANTIO DIRETO & $\begin{array}{l}11,2 \\
8\end{array}$ & 0,62 & 0,88 & 0 & 0 & 0 \\
\hline $\begin{array}{l}\text { PLANTIO } \\
\text { CONVENCIONAL }\end{array}$ & 2,40 & 1,04 & 0,99 & 41,43 & 1,05 & 0,98 \\
\hline $\begin{array}{l}\text { SISTEMA } \\
\text { NA TURAL }\end{array}$ & 5,15 & 0,92 & 0,93 & 33,68 & 0,99 & 0,96 \\
\hline $\begin{array}{l}\text { SOLO } \\
\text { SUBSUPERFICIAL }\end{array}$ & 2,42 & 1,02 & 0,98 & 41,40 & 1,07 & 0,98 \\
\hline
\end{tabular}

Segundo o critério do IBAMA (1990), os valores de $\mathrm{K}_{\mathrm{f}}$ classificam o solo dos diferentes sistemas testados como de baixa capacidade de adsorção. Valores baixos podem representar grande potencial de lixiviação do agrotóxico no perfil do solo e mesmo assim, os resultados demonstram comportamento distinto desse herbicida nos diferentes sistemas de manejo do solo.

Os valores de correlação $\left(\mathrm{r}^{2}\right)$ acima de 0,93 indicaram que as isotermas de adsorção dos solos sob SN, SC e SUB ajustaram-se à equação do modelo de Freundlich. O mesmo não foi observado no solo sob PD com $\mathrm{r}^{2}$ de 0,88 (Tabela 2). Esse comportamento também foi evidenciado pelo coeficiente $1 / \mathrm{n}$, cujo PD apresentou valor de 0,62 e os demais sistemas valores acima de 0,92. O parâmetro de Freundlich, 1/n, constitui medida de linearidade da isoterma de adsorção. Se for 
próximo a 1, a relação entre a quantidade adsorvida e a concentração de equilíbrio é linear e o cálculo do coeficiente de distribuição $K_{d}$ válido. O valor de $1 / n$ para PD indica que os dados não se ajustaram à equação de Freundlich.

Com exceção do solo sob PD que não se ajustou a equação, as isotermas de adsorção dos solos sob SN, SC e SUB apresentaram o mesmo comportamento descrito por GILES et al., 1960 (representado pelo modelo de partição de íons do Tipo-C). Segundo esses autores, o modelo indica constante afinidade do herbicida na solução com o solo como conseqüência da elevação de sítios disponíveis com o aumento do adsorbato (atrazina). Tal fato foi associado à presença de sítios restringidos como resultado da adsorção física e posterior difusão das moléculas através da microporosidade do solo (PIGNATELLO, 2000).

\subsection{DESSORÇÃO}

Os valores de $\mathrm{K}_{\mathrm{f}}$ das isotermas de dessorção (Figura 2) indicaram que o herbicida foi mais suscetível ao processo dessorção nos solos sob SC $\left(K_{f}=41,43\right)$ e SUB $\left(K_{f}=41,40\right)$ em relação ao $\mathrm{SN}\left(\mathrm{K}_{\mathrm{f}}=33,68\right)$. Isto significa o retorno do agrotóxico à solução do solo, disponibilizando o produto às perdas por lixiviação ao longo do tempo e contaminando as águas subterrâneas.

\section{FIGURA 2 - ISOTERMAS DE DESSORÇÃO DE ATRAZINA NO SOLO SOB PLANTIO DIRETO (PD), PLANTIO CONVENCIONAL (SC), SISTEMA NATURAL (SN) E SOLO SUBSUPERFICIAL (SUB)}

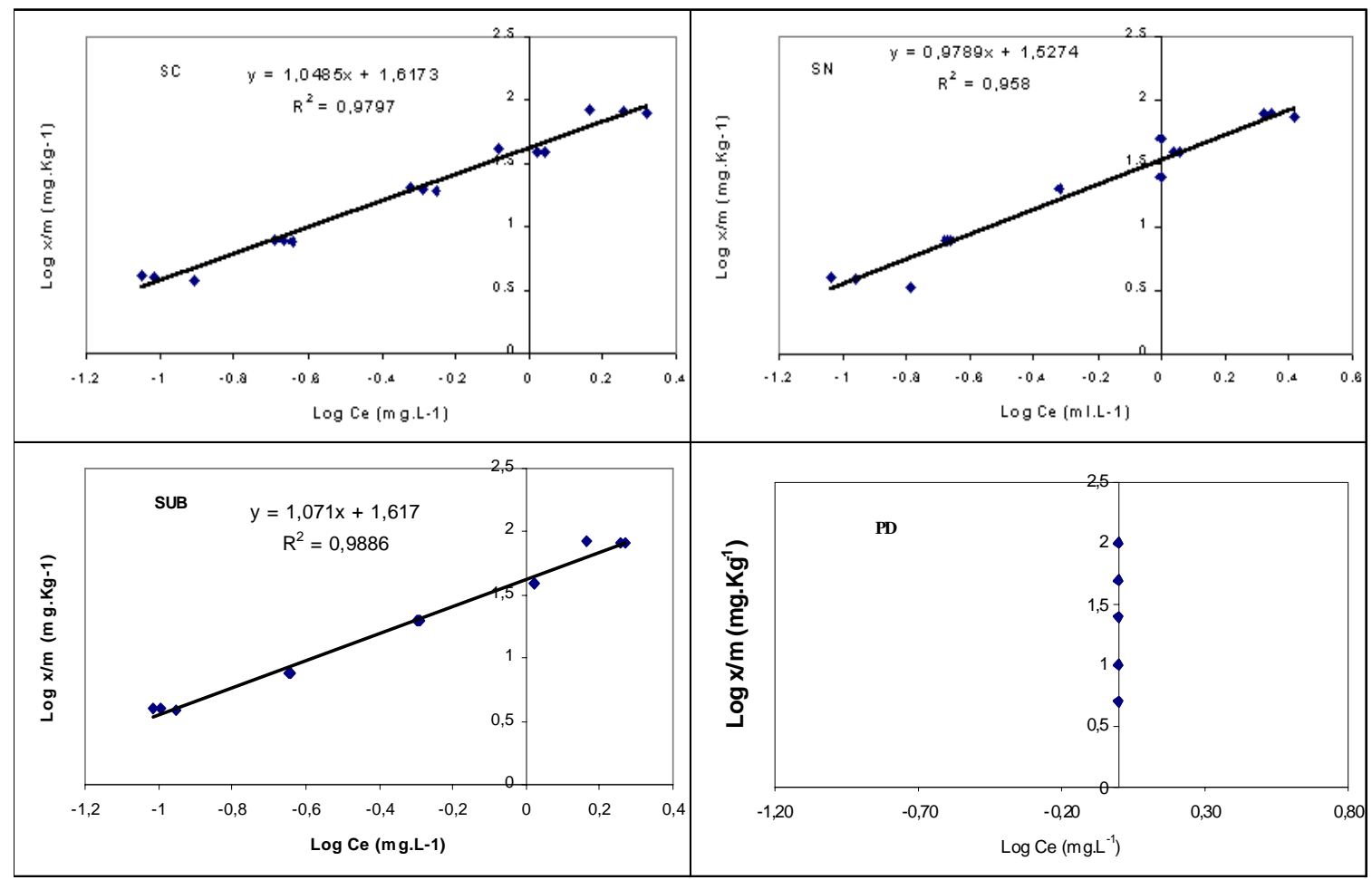

Cada ponto representa a média de três replicatas.

O solo sob PD apresentou valores de $\mathrm{K}_{\mathrm{f}}$ nas isotermas de dessorção igual a zero, mostrando que o processo de retorno do agrotóxico para solução do solo foi nulo. Embora existam diferentes tipos de ligação atrazina/solo (SENESI e TESTINI, 1982; PICCOLO e DE SIMONE, 1994), esses 
resultados sugerem que o processo de adsorção no solo submetido ao PD envolve energias de forte ligação. Esse comportamento foi observado por RODRIGUES, LIMA e YADA (2000), sendo verificada a influência do tipo de MO no processo de retenção. Se houvesse ligações de baixa energia, o processo de dessorção seria observado.

Os resultados de $r^{2}$ acima de 0,96 para os solos sob SN, SC e SUB demonstram que as isotermas de dessorção ajustaram-se a equação de Freundlich. Essa correlação também foi confirmada pelos valores de $1 / n$ nesses três solos (Tabela 2). O significado dos valores de $1 / n$ próximo a 1 mostram que não existem diferenças maiores quanto a energia dos sítios de adsorção nesses solos.

Os coeficientes $\mathrm{K}_{\mathrm{d}}$ obtidos para cada concentração do agrotóxico são apresentados na Tabela 3.

\section{TABELA 3 - VALORES DO COEFICIENTE DE ADSORÇÃO $\left(K_{\mathrm{D}}\right)$, COEFICIENTE DE ADSORÇÃO NORMALIZADO $\left(K_{O M}\right)$ E PERCENTAGEM DE ATRAZINA ADSORVIDA NO PD, SC, SN E SUB COM DIFERENTES CONCENTRAÇÕES DE ATRAZINA}

\begin{tabular}{|c|c|c|c|c|c|c|c|c|c|c|c|c|}
\hline & & PD & & & SC & & & SN & & & SUB & \\
\hline $\begin{array}{l}C_{i} \\
\mathrm{mg} \cdot \mathrm{L}^{-1}\end{array}$ & $K_{d}$ & $\%$ Ads & $\mathrm{K}_{\mathrm{om}}$ & $K_{d}$ & $\%$ Ads & $\mathrm{K}_{\mathrm{om}}$ & $K_{d}$ & $\%$ Ads & $\mathrm{K}_{\mathrm{om}}$ & $K_{d}$ & $\%$ Ads & $\mathrm{K}_{\mathrm{om}}$ \\
\hline 0,5 & 15,70 & 47,46 & 471,47 & 2,38 & 19,27 & 76,04 & 5,32 & 34,75 & 90,2 & 2,32 & 19,28 & 74,12 \\
\hline 1,0 & 15,07 & 69,07 & 452,55 & 2,36 & 19,33 & 75,40 & 5,86 & 36,97 & 89,56 & 2,33 & 19,33 & 74,44 \\
\hline 2,5 & 15,18 & 57,83 & 455,85 & 2,29 & 18,65 & 73,16 & 4,60 & 31,52 & 77,97 & 2,30 & 19,25 & 73,48 \\
\hline 5,0 & 8,26 & 51,73 & 248,04 & 2,60 & 20,67 & 83,06 & 5,10 & 33,80 & 86,44 & 2,55 & 20,55 & 81,47 \\
\hline 10 & 4,77 & 37,18 & 143,24 & 2,64 & 20,90 & 84,34 & 4,48 & 30,95 & 75,93 & 2,57 & 20,87 & 82,11 \\
\hline
\end{tabular}

O maiores valores de $\mathrm{K}_{\mathrm{d}}$, em média, foram encontrados no PD, seguido pelo $\mathrm{SN}$, SC e SUB para todas as concentrações estudadas. Esses refletem os valores da constante $K_{f} e$ também revelam a baixa retenção do herbicida no solo. No entanto, o PD apresentou maior potencial de adsorção em relação aos outros sistemas.

Os valores de $\mathrm{K}_{\mathrm{d}}$ para cada um dos solos sob SN, SC e SUB foram praticamente iguais nas diferentes concentrações. Já para o PD esses valores foram iguais nas concentrações de 0,5 a $2,5 \mathrm{mg} \mathrm{L}^{-1}$, havendo diminuição gradativa nas concentrações de 5 e $10 \mathrm{mg} \mathrm{L}^{-1}$ de atrazina. A redução desses valores, no entanto, não significou diminuição proporcional à quantidade de atrazina adsorvida ao solo (Tabela 3). A redução nos valores de $K_{d}$ no solo sob PD explica a falta de ajuste da equação de Freundlich para esse tipo de sistema. HANG (2002) observou flutuações nos valores de $K_{d}$ quando comparou solos com altos teores de $\mathrm{MO}$ e amostras de subsolo. Segundo LAIRD et al. (1994), o solo sob PD pode apresentar comportamento de flutuação devido à maior heterogeneidade da matriz do solo. Nos solos sob SC e SUB foram observados resultados de $\mathrm{K}_{\mathrm{d}}$ semelhantes, possivelmente, por se tratar de Latossolo com perfil de solo profundo e homogêneo (Tabela 3).

A Figura 3 apresenta a variação nos valores de $x / m$ em função do tempo de contato do herbicida com a fase sólida do solo. 


\section{FIGURA 3 - VARIAÇÃO DE C $\mathrm{S}_{\mathrm{S}}$ AO LONGO DO TEMPO NOS DIFERENTES SISTEMAS}

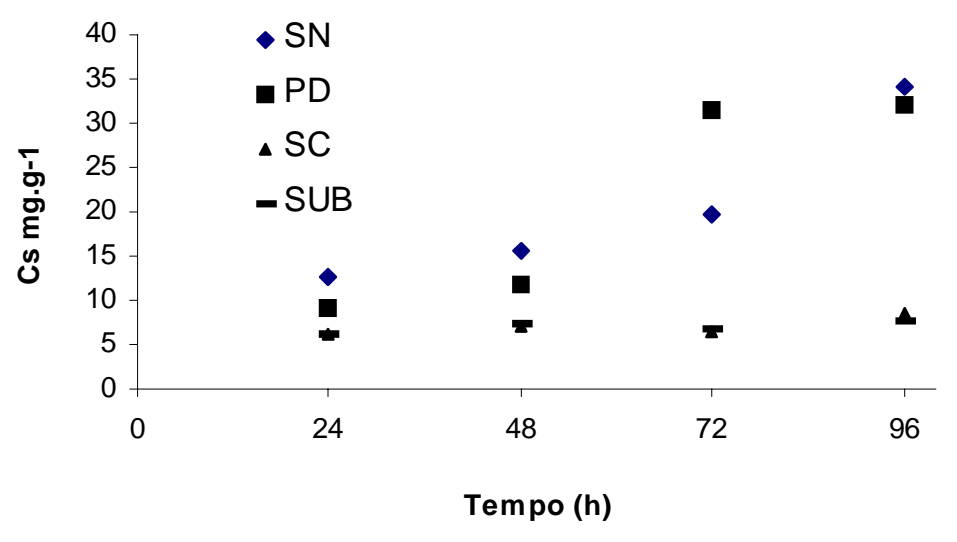

A maioria das metodologias para calcular o coeficiente de adsorção $\left(K_{d}\right)$ adota os tempos de 24 ou 48 horas para determinação de seus parâmetros. No entanto, o gráfico da Figura 3 mostra que os solos sob PD e SN apresentaram aumento crescente no valor de Cs da atrazina sem atingir equilíbrio até 96 horas. Já os solos sob SC e SUB apresentaram valores baixos, praticamente constantes em todos os tempos testados. Esses resultados confirmam a tendência ligeiramente crescente de adsorção da atrazina com o tempo de contato. Isto evidencia a existência de processos mais lentos a favor dessa adsorção, possivelmente por mecanismos de difusão dentro da matriz porosa (MYERS e WAUCHOPE, 1985) ou de absorção na fração orgânica (PIGNATELLO, 2000) nos solos sob PD e SN. Tal fato sugere que para PD e SN, a quantidade de herbicida presente na solução do solo decresce com o tempo e torna o produto menos disponível aos processos de lixiviação, volatilização e mineralização.

O coeficiente $\mathrm{K}_{\mathrm{om}}$, normalizado pelo teor de $\mathrm{MO}$ dos solos sob os diferentes sistemas é apresentado na Tabela 3. Os resultados respondem ao mesmo comportamento observado para os valores de $\mathrm{K}_{\mathrm{f}}$ e $\mathrm{K}_{\mathrm{d}}$, sendo encontrado maior potencial de adsorção de atrazina no solo PD (143 471), seguido pelo SN $(75,93$ - 90,2), SC $(73,16$ - 84,34) e SUB $(73,48$ - 82,11). Diversos autores sugerem que os valores dos parâmetros de adsorção dos agrotóxicos são diretamente proporcionais ao aumento do teor de matéria orgânica no solo. Esperava-se, então, que o SN (com maior teor de MO - $59 \mathrm{~g} \cdot \mathrm{Kg}^{-1}$ ) apresentasse maior potencial de adsorção. No entanto, esse comportamento não foi observado no presente estudo. O solo sob PD revelou maior potencial de adsorção, apresentando quase a metade do teor de $\mathrm{MO}$ de SN. Esses resultados apontam que não só a quantidade, mas a qualidade e o grau de humificação da MO incorporada ao solo devem ser considerados nos estudos de adsorção de agrotóxicos.

O estudo da qualidade da matéria orgânica pode fornecer melhores resultados quanto ao papel desse componente do solo na retenção de pesticidas. Essas diferenças de qualidade da MO do solo podem refletir os dados de PRATA (2002) em que a adsorção no PD e SC foi idêntica em ambos os sistemas. Embora o solo seja semelhante ao utilizado neste trabalho, os resultados controversos podem estar associados à diferença no sistema de rotação de culturas empregado.

Este trabalho mostrou que maior adsorção no solo PD sem dessorção constitui forma efetiva para redução do transporte de agrotóxicos para camadas mais profundas do solo, diminuindo o risco de contaminação das águas subterrâneas. O menor teor de matéria orgânica em PD, comparado ao SN, evidencia que a quantidade e a qualidade da MO são determinantes no processo de retenção. Outras pesquisas são necessárias para determinar se o fator responsável pela maior adsorção decorre da palha na superfície ou da decomposição da raíz que permanece no solo. 


\section{CONCLUSÃO}

As isotermas de adsorção de atrazina nos solos sob SC, SN e SUB ajustaram-se ao modelo de Freundlich. Para PD, o modelo não correspondeu ao comportamento do herbicida no solo devido flutuações nos resultados. A adsorção aumentou em função do tempo de contado da atrazina com os solos sob PD e SN, diminuindo os riscos de lixiviação com o decorrer do tempo. $O$ fator que promoveu maior potencial de adsorção no solo sob PD não foi exclusivamente o teor de $\mathrm{MO}$, mas a qualidade desse componente no solo. Maior potencial de adsorção e dessorção nula no sistema PD indicaram maior potencial na redução da lixiviação e contaminação dos lençóis d'água.

\section{ABSTRACT \\ ADSORPTION OF ATRAZINE IN TROPICAL SOIL UNDER NO-TILLAGE AND CONVENTIONAL TILLAGE}

The purpose of this research was to study the influence of a tillage system kind over the potential of atrazine's adsorption in the soil. The results of the adsorption coefficient showed that isotherms of atrazine in soils under conventional tillage (CT), natural system (SN) and subsuperficial soil (SUB) did fit to Freundlich model, while in the no-tillage (NT) the model did not answer to the behavior of the herbicide. Due the time that the atrazine was in contact with the PD and SN soil, the adsorption increased, reducing the leaching risks. The quality of the organic matter (OM) was, in fact, the reason that caused the largest potential of adsorption in the NT, and not only its quantity. The largest potential of adsorption and the null desorption in the NT, shows more chances of less leaching and water contamination. The main findings advises the NT as the technology that reduces environmental impact from the atrazine and other pesticides.

KEY-WORDS: SOIL POLUTION; LEACHING; HERBICIDE; ADSORPTION; NO-TILLAGE; ATRAZINE.

\section{REFERÊNCIAS}

1 AHMAD, R.; KOOKAMA, R.S.; ALSTON, A.M. Sorption of ametyn and imazethapyrin in twenty-five soils from Pakistan and Australia. Journal Environmental Science and Health A, v. 36, p. 143-160, 2001

2 BLANCO, F.M.G.; MACHADO, R. Persistence and leaching of atrazine and metolachlor in soil under corn. In: INTERNATIONAL WEED SCIENCE CONGRESS, 3., 2000, Foz do Iguaçu. Anais... Foz do Iguaçu: International Weed Science Society, 2000. p.90.

3 CORREIA, F.V.; MACRAE, A.; GUILHERME, L.R.G.; LANGENBACH, T. Atrazine sorption and fate in a Ultisol from humid tropical Brazil. Chemosphere, v.67, p. 847-854, 2007.

4 COSTA, M.A.; MONTEIRO, R.T.R.; TORNISIELO, V.L. Influência da adição de palha de cana-de-açúcar na degradação de 14C-Ametrina em solo areia quartzosa. Sciencia Agricola, Piracicaba, v. 54, p. 117-122, 1997.

5 ENTRY, A.J.; EMMINGHAM, W.H. The influence of dairy manure on atrazine and 2,4-dichlorophexyacetic acid mineralization on pasture soils. Canadian Journal of Soil Science, v. 25, p. 379-383, 1995.

6 FÜHR, F; OPHOFF, H.; BURAUEL, P.; WANNER, U.; HAIDER, K. Modification of the definition of bound residues. In: FÜHR, F; OPHOFF, H. Pesticide bound residues in soil. Weinheim: Wiley-VCH, 1998. p. 175-176.

7 GILES, C.H.; MACEWAN, T.H.; NAKHWA, S.N.; SMITH, D. Studies in adsorption. A system of classification of solution adsorption isotherms and use in diagnosis of adsorption mechanisms and in measurement of specific surface areas of solds. Journal Chemical Society, London, v. 11, p. 3974-3992, 1960.

8 HANCE, R.J. Adsorption and bioavailability. IN: GROVER, R. (Ed.) Environmental chemistry of herbicides. Boca Raton: CRC Press, 1988. cap.1, p.1-19.

9 HANG, Susana. Adsorción y degradación de atrazina en suelos de superficie y subsuelo de la provincial de Cordoba, Argentina. Córdoba, 2002.140f. Tese (Doutorado em Ciências Agropecuárias) - Faculdade de Ciências Agropecuárias -Universidade Nacional de Córdoba. 
10 IBAMA. Instituto Brasileiro de Meio Ambiente. Manual de testes para avaliação da ecotoxicidade de agentes químicos. 2. ed. Brasília, 1990. 351 p.

11 LAIRD, D.A.; YEN, P.Y.; KOSKINEN, W.C.; STEINHELMER, T.R.; DOWDY, R.H. Sorption of atrazine on soil clay components. Environmental Science \& Technology, v. 28; p. 1054-1061, 1994.

12 LANGENBACH, T.; SCHROLL, R.; SCHEUNERT, I. Fate of the herbicide ${ }^{14}$ Cterbuthylazine in Brazilian soils under various climatic conditions. Chemosphere, v. 45, p. 87-398, 2001.

13 LAVORENTI, A. Identificação de perigos de resíduos de pesticidas em substâncias húmicas. In: ENCONTRO BRASILEIRO DE SUBSTÂNCIAS HÚMICAS, 2., São Carlos. Anais... São Carlos: EMBRAPA, 1997. p. 66-71.

14 LUCHINI, L.C. Adsorptive behavior of herbicides in Brazilian soils. Arquivos do Instituto Biológico, v.64, n.1, p.43-49, 1997.

15 MYERS, R.S.; WAUCHOPE, R.D. Adsorption-desorption kinetics of atrazine and linuron in fresh water-sediment aqueous slurries. Journal Environmental Quality, v. 14, p. 132-136, 1985.

16 OECD guidelines for the testing of chemicals. Sorption/desorption using a batch equilibrium method OECE Test Guideline, 106. Paris, 2000

17 OLIVEIRA JÚNIOR, R.S. Relação entre propriedades químicas e físicas do solo e sorção, dessorção e potencial de lixiviação de herbicidas. Viçosa, 1998. 83 f. Tese (Doutorado em Fitotecnia) Universidade Federal de Viçosa.

18 PICOLLO, A.G.C.; DE SIMONE, C. Interaction of atrazine with humic substances of different origins and their hydrolyzed products. Science Total Environmental, v.117/118, p.403-412, 1994

19 PIGNATELLO, J. The measurement and interpretation of sorption and desorption rates for organics compounds in soil media. Advances in Agronomy, v.69, p.1-73, 2000.

20 PRATA, F; LAVORENTI, A.; REGITANO, J.B.; TORNISIELO, V.L. Degradação de sorção de ametrina em dois solos com aplicação de linhaça. Pesquisa Agropecuária Brasileira, v. 36, n. 7, p. 975-981, 2001.

21 PRATA, F. Comportamento do glifosato no solo e deslocamento miscível de atrazina. Piracicaba, 2002.149 f. Tese (Doutorado em Ciências), Centro de Energia Nuclear, Universidade de São Paulo.

22 RAO, P.S.C.; JESSUP, R.E. Sorption and movement of pesticides and other toxic organic substances in soils. In: KRAL, D.M. (Ed.) Chemical mobility and reactivity in soil systems. Madison, Wisconsin: Soil Science Society of America, 1983. Chap.13, p.183-201. (SSSA Special Publication, 11).

23 RODRIGUES, B.N.; LIMA, J.; YADA, I.F.U. Retenção pela palhada de herbicidas aplicados em pré-emergência na cultura do milho, em plantio direto. Revista Brasileira de Herbicidas, v. 1, n. 2, p. 123-128, 2000.

24 SENESI, N.; TESTINI, C. Physical-chemical investigations of interaction mechanisms between s-triazine herbicides and soil humic acids. Geoderma, v. 28, p. 129-146, 1982.

25 SILVA, R.F.; AQUINO, A.M.; MERCANTE, F.M.; GUIMARÃES, M.F. Macrofauna invertebrada do solo sob diferentes sistemas de produção em Latossolo da região do Cerrado. Pesquisa Agropecuária Brasileira, v. 41, n. 4, p. 697-704, 2006.

26 VAZ, C.M.P. Metodologia eletroanalítica para medida de atrazina em águas e solução de solos. Piracicaba, 1994. 97 f. Tese (Doutorado em Ciências), Centro de Energia Nuclear na Agricultura, Universidade de São Paulo.

\section{AGRADECIMENTOS}

Este trabalho contou com a valiosa colaboração do técnico Edir Martins Ferreira para as análises de resíduos e a Embrapa Agropecuária Oeste que criou as condições locais para os experimentos. Apoio financeiro obtido junto ao CNPq, FUJB, FAPERJ e PRONEX. 